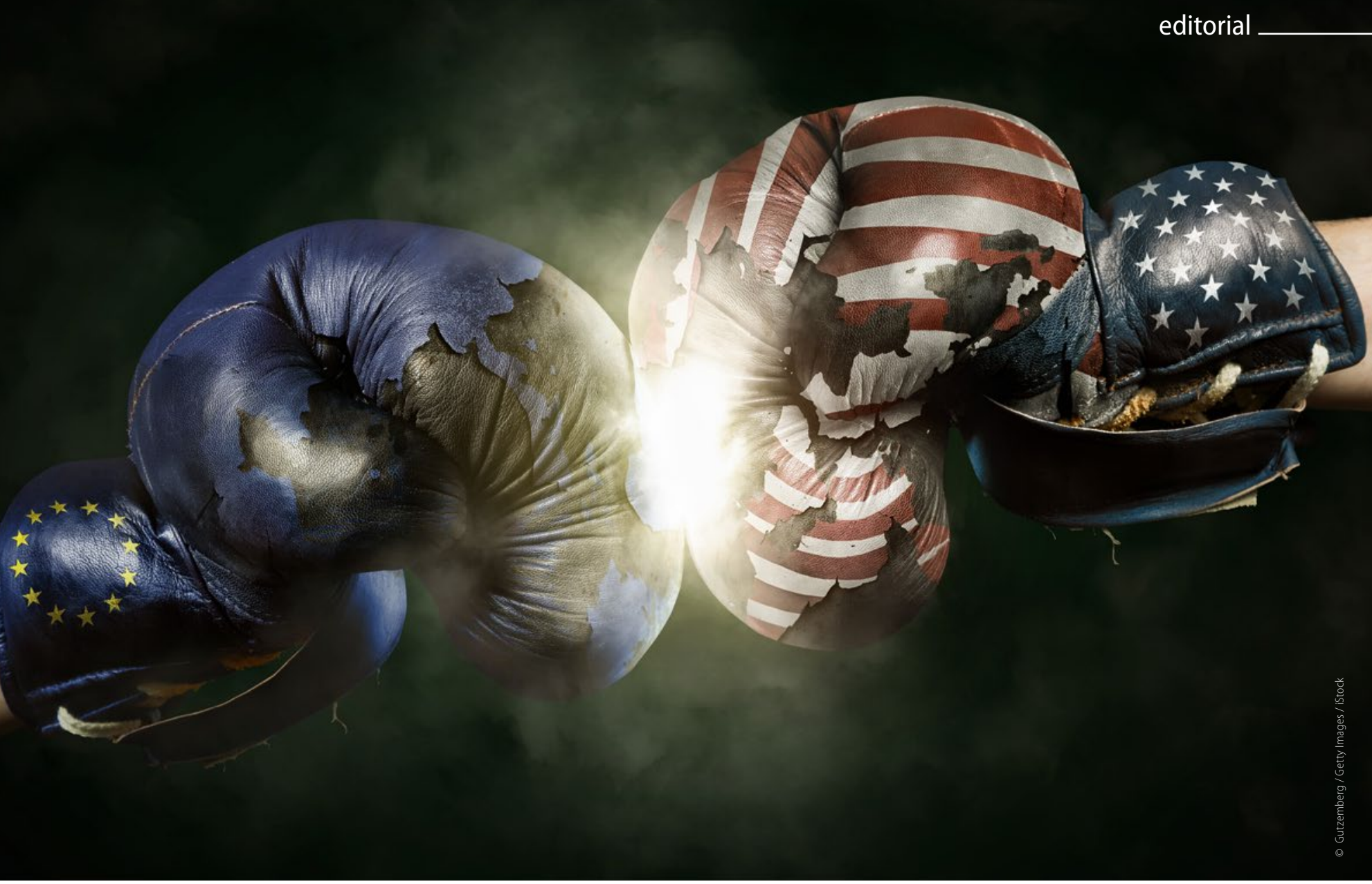

\title{
New World on Stage
}

Als die Wahlergebnisse in den Vereinigten Staaten bekannt wurden, hat es manchem Mitbürger schon gedämmert, dass mit der Amtsübernahme durch Donald Trump sich politisch nicht nur in den USA, sondern auch im Zusammenhang mit seinen Verbündeten, ja auf der gesamten Weltbühne etliches ändern würde. Wer dachte, dass Donald Trump nur leere Versprechungen macht, hat sich massiv geirrt. Innerhalb weniger Tage hat er seinen Ankündigungen Taten folgen lassen, die seine politische Stoßrichtung vorgeben: America first!

Die in Eintracht erstarrte deutsche Politik wird durch diese Amtsübernahme unsanft aus ihrem jahrzehntelangen Tiefschlaf und dem durch die GroKo induzierten Konsens geweckt. Die durch unsere Bundeskanzlerin verfolgte Politik muss sich nunmehr auch nach außen neu orientieren und im politischen Sturm in Europa und darüber hinaus bewähren.

Wichtigstes Thema ist die Wirtschaftspolitik, die durch den Austritt Großbritanniens aus der EU zusätzlich an Gewicht gewinnt. Die im Raum stehenden Strafzölle für den Exportweltmeister Deutschland lassen dunkle Wolken in unserer Handelsbilanz erahnen. Dabei ist dieser Handelsbilanzüberschuss teuer erkauft. Zum einen resultieren die Exportchancen aus einem niedrigen Euro, zum anderen aus jahrzehntelangem Sparen im Sozialbereich in Deutschland selber. Wir Ärzte können ein Lied davon singen, was es heißt, unter einem seit Jahrzehnten geltenden EBM wie auch unter einer aus 1983 stammenden GOÄ arbeiten zu müssen. Die Spitze in der Chronologie der Versäum-

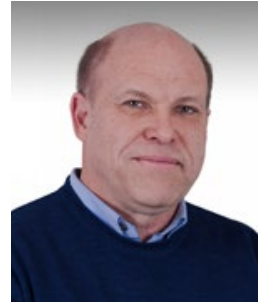

Dr. Matthias Herbst

Generalsekretär ADK e.V.

nisse ist dann noch der sogenannte Gesundheitsfonds, der 0,4\% Strafzinsen, also Millionen Euro an Versichertengeldern, in die Staatskasse zahlt. Weitere Summen in Milliardenhöhe wurden schon früher aus staatlichen Zuschüssen in andere Bereiche umgeleitet, wo Not am Mann war und ist!

Wenn also, wie in Hessen, Restzahlungen besser auf die Quartale verteilt werden, ist es nicht nur eine Freundlichkeit des Systems, sondern dieses versucht nun die Strafzinsen von $0,4 \%$ durch möglichst schnelles Weiterüberweisen des Geldes zu umgehen.

Eine realistische Sozialpolitik ist einer der Eckpunkte einer neuen Regierung. Ein Durchwursteln wie bisher muss endlich aufhören. Auch sehen wir in unseren Praxen die massiven Folgen der Zuwanderung. Gerade in Hessen erreichte uns dieser 
Tage ein Rundschreiben der Sozialverwaltung, die mit diesem endlich nach der von ihr selbst zugegebenen improvisierten Handlungsphase der Jahre 2015 und 2016 nunmehr ein Fundament für die weitere Behandlung von Migranten und Flüchtlingen durch den Aufbau einer entsprechenden Verwaltung schaffen will. Dazu muss man wissen, dass in Deutschland über 600 Sozialämter mit zum Teil unterschiedlichster Software zur Bewältigung des Ansturms ausgerüstet sind. Eine Sozial-Cloud einschließlich Fingerabdrücken zur Lösung dieses Problems scheint unbekannt (SAP wäre dankbar für einen derartigen Auftrag). Umgekehrt möchte unser Minister Gröhe aber eine zentrale Gesundheits-Cloud für seine Bürger einrichten, wie wir aus der FAZ vom 8. Januar 2017 erfuhren. Auch die weiteren Ausführungen sind eine Lektüre wert.

Es wundert einen nicht, wenn man bei jeder Gelegenheit erfährt, dass einige Zuwanderer verschiedene Identitäten verwandt haben, um mehrfach Flüchtlingshilfe in Anspruch zu nehmen. Laut Statistischem Bundesamt ist ein Jahresüberschuss von 1,1 Millionen Flüchtlingen für $2015 \mathrm{zu}$ verzeichnen. Wenn man davon ausgeht, dass 2016 rund 1 Million Menschen nach Deutschland gekommen sind, sind in den letzten zwei Jahren insgesamt 2,1 Million Menschen zugewandert. Inoffiziell dürfen noch einmal gut eine halbe Millionen Menschen dazu gerechnet werden, die ohne Registrierung eingesickert sind.

Für diese Menge an neuen Bürgern bedarf es natürlich besonderer Maßnahmen, um weiterhin gerechte und sichere Verhältnisse in Deutschland zu erhalten. Ohne ein modernes Einwanderungsgesetz, wie zum Beispiel dem kanadischen, kann es nicht gehen. Wir wollen in Deutschland gerade den kreativen Köpfen dieser Welt gerne ein zu Hause geben.

Dies muss durch ein Zuwanderungsgesetz begleitet werden. Flüchtlingen muss von vorne herein klar sein, dass sie unter allen Umständen mit ihren Papieren unterwegs sein sollten. Und: Wer einmal vor Gericht entsprechend verurteilt wird, verwirkt sein Asylrecht. Hierzu bedarf es einer klaren Regelung innerhalb der Europäischen Gemeinschaft und eines durchgehenden elektronischen Verifikationssystems. Man sollte doch in der EU in der Lage sein, all dies inklusive eines gemeinsamen Grenzschutzes in einer Zeit von sechs Monaten durchgehend zu schaffen. Einem Unternehmer und Populisten wie Trump sind solche Strukturen klar ein Dorn im Auge!

Somit bleibt die Frage, ob durch die 2015 erfolgte - humanitär verständliche - eher spontane Entscheidung von Angela Merkel, die Flüchtlinge ins Land zu lassen, sowohl den Brexit wie letztendlich auch den Wahlsieg von Donald Trump erst ermöglicht hat. Diese Frage muss jeder selbst beantworten. Eine andere Frage wäre, ob ein Bundeskanzler berechtigt ist, ohne Entscheidung des nationalen Parlaments und Abstimmung in der Europäischen Union derart weitreichende Entscheidungen zu treffen. Hierzu habe ich bis heute eigentlich keine vernünftige Antwort der Staatsrechtler gehört.

Was hat das alles mit den Wahlen in den USA und unserem Gesundheitswesen zu tun?

Ich bin fest davon überzeugt, dass in nächster Zeit ein stärkerer Wettbewerb als bisher ins Haus steht, zum einen ausgelöst durch eine entsprechende US-Wirtschaftspolitik inklusive der Zollschranken, zum anderen durch politische Verwerfungen wie dem ansteigenden Nationalismus in Europa samt Brexit. Reagieren wird die Regierung höchstwahrscheinlich wieder durch Einsparungen im Sozialbereich. Unsere Aufgabe ist es nun im Wahljahr 2017 auch in den eigenen Reihen jedem klarzumachen, dass ein "Weiterwursteln“" wie bisher nicht geht. Auch eine individuelle Gesundheitsleistung kann einmal zu Ende sein, spätestens dann wenn eine Gebührenordnung diese nicht mehr vorsieht oder die privaten Patienten ganz abgeschafft werden. Folgt man einer Studie der Bertelsmann-Stiftung, insbesondere was die Beihilferegelung und deren Kosten im öffentlichen Dienst angeht, lassen sich mit einer Abschaffung dieser Regelung und einer Überführung der Patienten in die GKV Millionen einsparen. Es ist also dringend notwendig, entsprechende Strategien zu entwickeln, um variabel auf die bevorstehenden Herausforderungen zu reagieren.

Die Begeisterung für Martin Schulz unter der Überschrift „Gerechtigkeit für alle “, zum Beispiel die Ungerechtigkeiten des Steuersystems in Deutschland zu beseitigen, kann recht schnell zu einer generellen Abkehr der Bürger auch vom bestehenden Gesundheitssystem führen. Madig wird es von der Politik seit Jahren gemacht. Effizient ist es ehrlicherweise nicht. Die Bürokratie spricht Bände! Es ist keineswegs sicher, wer im Herbst die Wahl wirklich gewinnt und wer dann wie seine Schwerpunkte in welcher Koalition setzt.

Nach 70 Jahre Frieden in Europa wäre es wunderbar, wenn die nächsten 30 Jahre ebenfalls von Frieden und Verständigung untereinander nach innen und nach außen gekennzeichnet wären. Und wie wäre es denn, wenn sich die Politik einmal für die oft unentgeltliche Zuwendung der vielen Akteure im Gesundheitswesen bedanken würde, die geholfen haben, die humanitären Auswirkungen dieser Zuwanderung in unermüdlicher Arbeit zu bewältigen?

Mit freundlichen Gruß

Ihr

Dr. Matthias Herbst

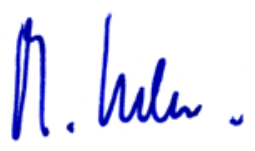

